$\begin{array}{lllll}\text { April } & 2013 & 10: 48 & \text { WSPC/INSTRUCTION } & \text { FILE }\end{array}$

MajPolytope 'DahlZhang'Final'20130329

Discrete Mathematics, Algorithms and Applications

(C) World Scientific Publishing Company

\title{
INTEGRAL MAJORIZATION POLYTOPES
}

\author{
GEIR DAHL \\ Department of Mathematics, CMA, University of Oslo \\ P.O. Box 1053, Blindern, 0316 Oslo, Norway \\ geird@math.uio.no \\ FUZHEN ZHANG \\ Farquhar College of Arts and Sciences, Nova Southeastern University \\ 3301 College Ave., Fort Lauderdale, Florida 33314, USA \\ zhang@nova.edu
}

Received Day Month Year

Accepted Day Month Year

\begin{abstract}
The majorization polytope $M(a)$ consists of all vectors dominated (or majorized, to be precise) by a given vector $a \in \mathbb{R}^{n}$; this is a polytope with extreme points being the permutations of $a$. For integral vector $a$, let $\nu(a)$ be the number of integral vectors contained in $M(a)$. We present several properties of the function $\nu$ and provide an algorithm for computing $\nu(a)$.
\end{abstract}

Keywords: Ferrers diagram; integer partition; majorization; polytope.

Mathematics Subject Classification 2000: 52B05; 05A17; 15A39

\section{Introduction}

Let $n$ be a positive integer. We may write (or split) $n$ as sums of $n$ nonincreasing nonnegative integers $p_{1}, p_{2}, \ldots, p_{n}$ in different ways (or partitions; see Section 2). For example, $3=3+0+0=2+1+0=1+1+1$. If we denote by $P(n)$ the number of different partitions of $n$, then $P(3)=3$. One may check that $P(5)=7$. As $n$ gets large, $P(n)$ increases rapidly. It is astounding that $P(200)$ is about 4 trillion [1, p. 68]. The determination of $P(n)$ is an intriguing and difficult problem in number theory and combinatorics (see, e.g., [12] and [9, Chapter 15]). It has much to do with the theories of majorization and polytopes. In the language of majorization, $P(5)=7$ means that there are 7 nonincreasing integral vectors in $\mathbb{R}^{5}$ that are majorized by the vector $(5,0,0,0,0)$. Equivalently, there are 7 nonincreasing integral vectors in $\mathbb{R}^{5}$ that are contained in the majorization polytope generated by $(5,0,0,0,0)$. In this paper we study majorization polytopes for more general integral vectors.

For vectors $x$ and $a$ in $\mathbb{R}^{n}$, we say that $x$ is majorized by $a$, denoted by $x \preceq a$, 


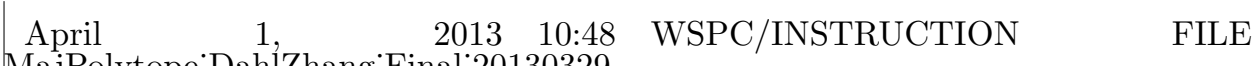

MajPolytope'DahlZhang'Final'20130329

provided that $\sum_{j=1}^{k} x_{[j]} \leq \sum_{j=1}^{k} a_{[j]}$ for $k=1,2, \ldots, n$, where there is an equality for $k=n$. Here $x_{[j]}$ is the $j$ th largest component of $x, j=1,2, \ldots, n$. Roughly speaking, that $x$ is majorized by $a$ means that the components of $x$ are dominated by or less "spread-out" than the components of $a$. For given $a \in \mathbb{R}^{n}$, the majorization polytope $M(a)$ is the collection of all vectors majorized by $a$, that is,

$$
M(a)=\left\{x \in \mathbb{R}^{n}: x \preceq a\right\} .
$$

We remark that $M(a)$ is known under different names in the literature, e.g., "permutohedron" (see [10]) or "permutation polytope" a (see [2], where facial properties of this polytope are presented). Our $M(a)$ may also be interpreted as the convex hull conv $S_{n}(a)$ of the set $S_{n}(a)$ consisting of all permutations of vector $a$.

Majorization theory was first formally introduced in the Hardy-LittlewoodPólya's well known book Inequalities [7, p. 45]. The monograph [8] contains a comprehensive study of majorization and its applications. Also, [3] treats majorization in connection with several combinatorial classes of matrices. In [14] majorization is discussed in detail in connection with matrix theory, particularly the spectral properties of matrices, etc.

Let $M_{I}(a)$ be the set of all integral vectors (i.e., all components are integers) contained in $M(a)$. We are interested in the cardinality of $M_{I}(a)$ and its dependence on $a$. As we shall see, the cardinality $\nu(a)$ of $M_{I}(a)$ is closely related to integer partitions. In Section 2 we show several properties of the function $a \rightarrow \nu(a)$, and in Section 3 we introduce an operation splitting and a recursive algorithm for computing $\nu(a)$ based on operations on Ferrers diagrams.

\section{Properties of $\nu$ and $\nu^{*}$}

As usual, the $j$ th unit vector of $\mathbb{R}^{n}$ is denoted by $e_{j}$, i.e., $e_{j}$ has $j$ th component 1 and 0 elsewhere. For a vector $x=\left(x_{1}, x_{2}, \ldots, x_{n}\right) \in \mathbb{R}^{n}$, its $j$ th component is $x_{j}$. Let $x_{[j]}$ denote its $j$ th largest component: $x_{[1]} \geq x_{[2]} \geq \cdots \geq x_{[n]}$ and write $x^{\downarrow}=\left(x_{[1]}, x_{[2]}, \ldots, x_{[n]}\right)$. We say that $x \in \mathbb{R}^{n}$ is monotone if $x_{1} \geq x_{2} \geq \cdots \geq x_{n}$. So for any $x \in \mathbb{R}^{n}, x^{\downarrow}$ is monotone.

We consider the sets $M(a)$ and $M_{I}(a)$ as defined in Section 1 . Note that $M(a)=$ $M\left(a^{\prime}\right)$ when $a^{\prime}$ is a permutation of $a$. For $a \in \mathbb{R}^{n}$, define

$$
\nu(a)=\left|M_{I}(a)\right| .
$$

So $\nu(a)$ is the number of integral vectors majorized by $a$. Let $M_{I}^{*}(a)$ denote the set of monotone vectors in $M_{I}(a)$, and define

$$
\nu^{*}(a)=\left|M_{I}^{*}(a)\right| .
$$

Our goal is to investigate the functions $\nu$ and $\nu^{*}$. Apparently, $\nu(a)$ equals zero if the sum of the components of $a$ is not an integer. For example, $a=\left(2, \frac{1}{2}\right), \nu(a)=0$.

aNote: the term "permutation polytope" sometimes refers to a different object, namely, the convex hull of a group of permutation matrices. 
We assume that the vectors in the study are integral. From the previous section, if $a=(5,0,0,0,0)$, we know that $\nu^{*}(a)=7$.

Example 2.1. Let $a=(4,2,1)$. Then $\nu(a)=12$ and $\nu^{*}(a)=3$. In fact, $M_{I}(a)$ consists of: the six permutations of $(4,2,1)$, along with the three (different) permutations of $(3,3,1)$ and the three permutations of $(3,2,2) . M(a)$ is the convex hull of the permutations of $(4,2,1)$.

If $a$ is a constant vector (i.e., all components are equal), then $x \preceq a$ implies $x=a$, so $M_{I}(a)=M_{I}^{*}(a)=\{a\}$ and thus $\nu(a)=\nu^{*}(a)=1$. In general, for an integral $a \in \mathbb{R}^{n}$, since every vector in $M_{I}^{*}(a)$ generates through permutation at most $n$ ! vectors in $M_{I}(a)$, we have $\nu(a) \leq \nu^{*}(a) n$ !. In addition, $\nu(-a)=\nu(a)$ and $\nu^{*}(-a)=\nu^{*}(a)$. For integral $a=(\alpha, \beta) \in \mathbb{R}^{2}$ with $\alpha \geq \beta$, we observe that $\nu(a)=\alpha-\beta+1$ and $\nu^{*}(a)=\left\lfloor\frac{\alpha-\beta}{2}\right\rfloor+1$.

The following result from [6] will be useful to prove our Proposition 2.3.

Theorem 2.2. ([6]) Let $a, b \in \mathbb{R}^{n}$ be monotone vectors. Then $M(a+b)=M(a)+$ $M(b)$. If, in addition, $a$ and $b$ are integral, then $M_{I}(a+b)=M_{I}(a)+M_{I}(b)$. (Here $S+T=\{s+t: s \in S, t \in T\}$.)

Below are some basic observations about the function $\nu$.

Proposition 2.3. Let $a, b \in \mathbb{R}^{n}$ be integral vectors. Then the following hold:

(i) If $a \preceq b$, then $\nu(a) \leq \nu(b)$.

(ii) If $a$ is a constant vector, then $\nu(a+b)=\nu(b)$.

(iii) $\nu(a+b) \leq \nu(a) \nu(b)$. Equality holds if and only if $a$ or $b$ is a constant vector.

(iv) $\nu(k a) \leq \nu^{k}(a)$ for any positive integer $k$. Equality occurs if and only if $k=1$ or $a$ is a constant vector.

Proof. (i). The majorization order is transitive. So $a \preceq b$ implies that $M_{I}(a) \subseteq$ $M_{I}(b)$. The cardinality inequality follows immediately.

(ii). If $a$ is a constant vector, then $x \preceq b$ if and only if $a+x \preceq a+b$. There is a bijection between $M_{I}(b)$ and $M_{I}(a+b)$. So $\nu(a+b)=\nu(b)$.

(iii). Note that $a+b \preceq a^{\downarrow}+b^{\downarrow}$. It follows that $\nu(a+b)=\left|M_{I}(a+b)\right| \leq$ $\left|M_{I}\left(a^{\downarrow}+b^{\downarrow}\right)\right|=\left|M_{I}\left(a^{\downarrow}\right)+M_{I}\left(b^{\downarrow}\right)\right| \leq \nu(a) \nu(b)$ (the second equality is by Theorem 2.2). Assuming that $a$ and $b$ are not constant vectors, we show that the strict inequality holds. To this end, it suffices to show that $M_{I}(a)+M_{I}(b)$ contains at least one duplicated element. Since $a$ and $b$ are non-constant integral vectors, there are permutations $a^{\prime}$ and $b^{\prime}$ of $a$ and $b$, respectively, with $a^{\prime}=(\alpha, \beta, \ldots), \alpha>\beta$, and $b^{\prime}=(p, q, \ldots), p<q$. Set $\tilde{a}=(\alpha-1, \beta+1, \ldots)$ and $\tilde{b}=(p+1, q-1, \ldots)$, where $\tilde{a}$ and $\tilde{b}$ have the same remaining components as $a^{\prime}$ and $b^{\prime}$, respectively. Then $\tilde{a} \preceq a$ and $\tilde{b} \preceq b$. Apparently, $\tilde{a} \neq a^{\prime}$ and $\tilde{b} \neq b^{\prime}$. However, $a^{\prime}+b^{\prime}=\tilde{a}+\tilde{b}$.

(iv). This is a consequence (repeated use) of (iii) by setting $a=b$. 
$\begin{array}{lllll}\text { April } & 1, \quad 2013 \quad 10: 48 & \text { WSPC/INSTRUCTION } & \text { FILE }\end{array}$

MajPolytope 'DahlZhang 'Final'20130329

Remark. Regarding (i), one may show that strict inequality holds if $a$ is not a permutation of $b$. Furthermore, $\nu^{*}(a) \leq \nu^{*}(b)$, with equality if and only if $a$ is a permutation of $b$. We also point out that these inequalities do not generalize to weak majorizations. Property (ii) reveals that the cardinality of $M_{I}(b)$ remains unchanged through "shifting". Thus the vectors may be assumed to be nonnegative. In addition, if $a$ is a constant vector, then $\nu^{*}(a+b)=\nu^{*}(b)$. The analogous result of (iii) for $\nu^{*}$, i.e., $\nu^{*}(a+b) \leq \nu^{*}(a) \nu^{*}(b)$, does not hold in general. For example, take $a=b=(1,0)$. Then $\nu^{*}(a)=\nu^{*}(b)=1$, however, $\nu^{*}(a+b)=2$.

Given $v \in M_{I}^{*}(a)$, let $v$ have $k$ distinct components $\tilde{v}_{1}>\tilde{v}_{2}>\cdots>\tilde{v}_{k}, 1 \leq k \leq$ $n$, and let $\tilde{v}_{i}$ occur $n_{i}$ times in $v$. So $n_{1}+\cdots+n_{k}=n$. Denote

$$
\kappa(v)=\frac{n !}{n_{1} ! \cdots n_{k} !} .
$$

Proposition 2.4. Let $a \in \mathbb{R}^{n}$. Then

$$
\nu(a)=\sum_{v \in M_{I}^{*}(a)} \kappa(v) .
$$

Proof. This is because each $v$ in $M_{I}^{*}(a)$ generates $\kappa(v)$ vectors in $M_{I}(a)$.

Example 2.5. Let $a=(4,2,1)$. Then $M_{I}^{*}(a)=\{a, u, v\}$, where $u=(3,3,1)$, $v=(3,2,2)$. Moreover, $\kappa(a)=6, \kappa(u)=6 / 2=3, \kappa(v)=6 / 2=3$, so $\nu(a)=$ $6+3+3=12$ as we found in Example 2.1.

Corollary 2.6. If $a=(s+t, \ldots, s+t, s, \ldots, s) \in \mathbb{R}^{n}$, where the first $k(1 \leq k<n)$ components are $s+t$, for some integer $s$ and positive integer $t$, then

$$
\nu(a) \leq\left(\begin{array}{l}
n \\
k
\end{array}\right)^{t} .
$$

Equality holds if and only if $t=1$.

Proof. Write $a=s e+t(1, \ldots, 1,0, \ldots, 0)$, where $e$ is the all-ones vector. By Proposition 2.3 (ii) and (iv), we have $\nu(a)=\nu(t(1, \ldots, 1,0, \ldots, 0)) \leq\left(\begin{array}{l}n \\ k\end{array}\right)^{t}$. Equality holds if and only if $t=1$ because $a$ is not a constant vector.

For the equality $(t=1)$ case, alternatively, the only vectors majorized by $a$ are the permutations of $a$. Any such permutation of $a$ corresponds to a selection of the $k$ positions containing $s+1$, and the number of such selections is $\left(\begin{array}{l}n \\ k\end{array}\right)$. One can also see this from Proposition 2.4: $\nu(a)=\kappa(a)=\frac{n !}{k !(n-k) !}=\left(\begin{array}{l}n \\ k\end{array}\right)$.

The cardinality functions $\nu$ and $\nu^{*}$ are related to integer partitions. A partition of a positive integer $n$ is a nonincreasing sequence $p_{1}, p_{2}, \ldots, p_{k}$ of positive integers whose sum is $n$. (We may add trailing zeros for convenience.) Clearly, such a partition may be represented by a monotone integral vector $\left(p_{1}, p_{2}, \ldots, p_{k}\right)$ (with the 
correct sum of its components). Each $p_{i}$ is a part of the partition. Let $\mathcal{P}(n)$ be the set of all partitions of $n$ (a subset of $\mathbb{R}^{n}$ ) and denote the number of partitions of an integer $n$ by $P(n)$. So $P(n)=|\mathcal{P}(n)|$. It has been evident that determination of $P(n)$ is an intriguing and difficult problem in number theory and combinatorics; see [12] and [9, Chapter 15] for related results in this area. We observe that $\mathcal{P}(n)$ coincides with $M_{I}^{*}(a)$ when $a=(n, 0, \ldots, 0) \in \mathbb{R}^{n}$. Thus integer partition may be described and studied by means of majorization.

Proposition 2.7. Let $a=(n, 0, \ldots, 0) \in \mathbb{R}^{n}$. Then

$$
\nu^{*}(a)=P(n), \quad \nu(a)=\sum_{p \in \mathcal{P}(n)} \kappa(p)=\sum_{\substack{n \geq p_{1}>\cdots>p_{q} \geq 1 \\ n_{1} p_{1}+\cdots+n_{q} p_{q}=n}} \frac{n !}{n_{1} ! \cdots n_{q} !\left(n-\sum_{i=1}^{q} n_{i}\right) !} .
$$

Proof. The first part is obvious because $M_{I}^{*}(a)$ contains exactly the partitions of $n$, that is, $M_{I}^{*}(a)$ coincides with $\mathcal{P}(n)$. The second part follows from Proposition 2.4. Note that in a partition $n=p_{1}+\cdots+p_{1}+\cdots+p_{q}+\cdots+p_{q}+0+\cdots+0$, $p_{1}>\cdots>p_{q} \geq 1$, each $p_{i}$ appears $n_{i}$ times, 0 appears $n-n_{1}-\cdots-n_{q}$ times.

A classical result of Euler (see, e.g., [9, p. 155] or [12, p. 7]) gives the generating function of $P(n)$ (in the summation below we define $P(0)=1$ ):

$$
\begin{aligned}
\sum_{n=0}^{\infty} P(n) x^{n} & =\frac{1}{1-x} \cdot \frac{1}{1-x^{2}} \cdot \frac{1}{1-x^{3}} \cdots \\
& =\left(1+x+x^{2}+\cdots\right)\left(1+x^{2}+x^{4}+\cdots\right)\left(1+x^{3}+x^{6}+\cdots\right) \cdots .
\end{aligned}
$$

It is possible to compute the numbers $P(n)$ recursively. Define $P_{k}(n)$ as the number of partitions of $n$ into $k$ parts. This is the same as the number of integral solutions of $x_{1}+x_{2}+\cdots+x_{k}=n, x_{1} \geq x_{2} \geq \cdots \geq x_{k} \geq 1$, which again equals the number of integral solutions of $z_{1}+z_{2}+\cdots+z_{k}=n-k, z_{1} \geq z_{2} \geq \cdots \geq z_{k} \geq 0$. Considering the number of these $z_{i}$ 's that are 1 reveals the recursion $([9$, p. 152])

$$
P_{k}(n)=\sum_{s=1}^{k} P_{s}(n-k) \quad(1 \leq k \leq n-1),
$$

with $P_{1}(n)=P_{n}(n)=1$ for all $n$ and $P_{k}(n)=0$ when $k>n$. This makes it possible to compute the $P_{k}(n)$ 's efficiently. Finally, one may compute $P(n)$ by $P(n)=\sum_{k=1}^{n} P_{k}(n)$. For instance, if we view the numbers $P_{k}(n)$ as the $(k, n)$ entry of a matrix $P$, this matrix may be computed row by row, and its column sums are the numbers $P(1), P(2), P(3), \ldots$ Although no explicit formula for $P(n)$ is known, several estimates are available; see, e.g., [12] and [9, Chapter 15].

Given a positive integer $n$, the number of ways that $n$ is written as a sum of at most $m$ parts can be described by the function $\nu$ and such function is bounded by $m^{n}$. To see this, let $(n, 0, \ldots, 0) \in \mathbb{R}^{m}$ and write $a=n(1,0, \ldots, 0)$. By Proposition 2.3 (iv), we have $\nu(a) \leq \nu^{n}(1,0, \ldots, 0)=m^{n}$. 
Proposition 2.8. For positive integers $m, n$, let $a=(n, 0, \ldots, 0) \in \mathbb{R}^{m}$. Then

$$
\nu^{*}(a)=\sum_{k=1}^{\min \{m, n\}} P_{k}(n) ; \quad \nu(a) \leq \sum_{k=1}^{\min \{m, n\}} \frac{m !}{(m-k) !} P_{k}(n) .
$$

Proof. The identity for $\nu^{*}(a)$ follows from the aforementioned discussions. For $\nu(a)$, it is sufficient to notice that each element $\left(p_{1}, \ldots, p_{k}, 0, \ldots, 0\right) \in \mathbb{R}^{m}$ counted in $P_{k}(n)$ can generate at most $\frac{m !}{(m-k) !}$ vectors in $M_{I}(a)$.

Example 2.9. Let $a=(5,0,0) \in \mathbb{R}^{3}, n=5, m=3$. Then

$$
\sum_{k=1}^{\min \{m, n\}} \frac{m !}{(m-k) !} P_{k}(n)=\sum_{k=1}^{3} \frac{3 !}{(3-k) !} P_{k}(5)=27 \text {. }
$$

Example 2.10. Let $a=(3,0,0,0,0) \in \mathbb{R}^{5}, n=3, m=5$. Then

$$
\sum_{k=1}^{\min \{m, n\}} \frac{m !}{(m-k) !} P_{k}(n)=\sum_{k=1}^{3} \frac{5 !}{(5-k) !} P_{k}(3)=85 .
$$

The following result gives an upper bound for $\nu(a)$ in terms of $m$ and $n$. This bound, not necessarily the best, but we believe, is better than $m^{n}$ (that we discussed prior to Proposition 2.8). However, no proof is available yet.

Corollary 2.11. For positive integers $m, n$, let $a=(n, 0, \ldots, 0) \in \mathbb{R}^{m}$. Then

$$
\nu(a) \leq \sum_{k=1}^{\min \{m, n\}}\left(\begin{array}{c}
m \\
k
\end{array}\right)\left(\begin{array}{c}
n+\frac{k(k-1)}{2}-1 \\
k-1
\end{array}\right)
$$

Proof. It is known $\left[9\right.$, p. 154] that $k ! P_{k}(n) \leq\left(\begin{array}{c}n+\frac{k(k-1)}{2}-1 \\ k-1\end{array}\right)$. So

$$
\frac{m !}{(m-k) !} P_{k}(n)=\left(\begin{array}{c}
m \\
k
\end{array}\right) k ! P_{k}(n) \leq\left(\begin{array}{c}
m \\
k
\end{array}\right)\left(\begin{array}{c}
n+\frac{k(k-1)}{2}-1 \\
k-1
\end{array}\right) .
$$

The upper bound is immediate from Proposition 2.8.

Proposition 2.12. Let $a=\left(a_{1}, a_{2}, \ldots, a_{n}\right)$ be an integral vector. Then

$$
\nu(a) \leq \min \left\{n^{\sum_{k=1}^{n}\left|a_{k}\right|}, \prod_{k=1}^{n}\left(\sum_{t=1}^{\min \left\{n,\left|a_{k}\right|\right\}} \frac{n !}{(n-t) !} P_{t}\left(\left|a_{k}\right|\right)\right)\right\} .
$$


Proof. Write $a=a_{1} e_{1}+a_{2} e_{2}+\cdots+a_{n} e_{n}$. By Proposition 2.3 (iii), we have

$$
\nu(a) \leq \prod_{k=1}^{n} \nu\left(a_{k} e_{k}\right)=\prod_{k=1}^{n} \nu\left(\left|a_{k}\right| e_{k}\right) \leq \prod_{k=1}^{n}\left(\sum_{t=1}^{\min \left\{n,\left|a_{k}\right|\right\}} \frac{n !}{(n-t) !} P_{t}\left(\left|a_{k}\right|\right)\right) .
$$

The last inequality is by Proposition 2.8. On the other hand, by Proposition 2.3 (iv), we have for each $k$,

$$
\nu\left(a_{k} e_{k}\right)=\nu\left(\left|a_{k}\right| e_{k}\right) \leq n^{\left|a_{k}\right|} .
$$

Thus $\nu(a) \leq n^{\sum_{k=1}^{n}\left|a_{k}\right|}$. Combining these reveals the desired inequality.

\section{The splitting operation}

Let $a=\left(a_{1}, a_{2}, \ldots, a_{n}\right)$ be a nonnegative integral vector in $\mathbb{R}^{n}$ and let $N=\sum_{j=1}^{n} a_{j}$. Then each vector in $M_{I}^{*}(a)$ is a partition of $N$ "controlled" by $a$. In this section, we study partitions using Ferrers diagrams (or Young diagrams). For instance, the partition $p=(5,4,1)$ of $N=10$ corresponds to the Ferrers diagram

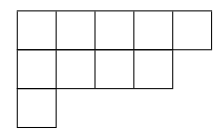

in which the number of boxes (squares) in the first row is the first part $p_{1}=5$, etc.

Let $N$ and $n$ be positive integers and let $\mathcal{P}_{N, n}$ be the set of all monotone integral vectors of length $n$ whose sum of components equals $N$. This corresponds to partitions of $N$ into at most $n$ parts. Then $\mathcal{P}_{N, n}$ equipped with majorization ordering becomes a partially ordered set (poset) which has been studied in, e.g., [4]. This poset has a unique maximal element $(N, 0, \ldots, 0) \in \mathbb{R}^{n}$ and a unique minimal element $(v+1, \ldots, v+1, v, \ldots, v) \in \mathbb{R}^{n}$, where $v=\lfloor N / n\rfloor$ and the number of components being $v+1$ is $N-n v$.

Given a monotone integral vector $a \in \mathbb{R}^{n}$, the set $M_{I}^{*}(a)$ is a subset of the poset $\mathcal{P}_{N, n}$, where $N=\sum_{j=1}^{n} a_{j}$, so $M_{I}^{*}(a)$ is a subposet. It is actually the principal ideal in $\mathcal{P}_{N, n}$ generated by $a$ (see, e.g., [5] or [11, Chapter 3]). The set $M_{I}^{*}(a)$, or rather the corresponding Ferrers diagrams, may be constructed recursively as follows: Start with the Ferrers diagram of $a$ and, repeatedly, choose a box at the end of a row and move it to the end of some row below, assuming monotonicity of the parts is preserved. Moving a box in this way corresponds to an integral transfer and it is known that any integral vector majorized by $a$ may be produced by a sequence of such transfers (see [8, Chapter 5]). An enumeration like this is, of course, only practical for computing $\nu^{*}(a)$ when this number is reasonably small. A better (more efficient) approach is introduced in this section. That is, instead of moving one box each time, we move multiple boxes each time by so-called splitting vectors that are less spread-out and have lower dimensions. 
Example 2.1 - continued. Let again $a=(4,2,1)$. Then $M_{I}^{*}(a)$ contains 3 vectors and their Ferrers diagrams are
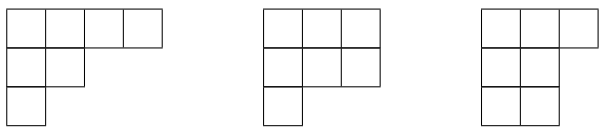

Now we introduce an operation on monotone integral vectors which is convenient to explain using Ferrers diagrams. Let $F$ be the Ferrers diagram of a monotone integral vector $a=\left(a_{1}, a_{2}, \ldots, a_{n}\right)$, define $N=\sum_{j=1}^{n} a_{j}$, and consider an integer $j$ with $\lceil N / n\rceil \leq j \leq a_{1}$. Let $F^{j \mid}$ be the Ferrers diagram obtained from $F$ by moving all boxes in columns $j+1, j+2, \ldots, a_{1}$ to other rows with preference to the uppermost rows, and then deleting the first row. (If $j=a_{1}$, no boxes are moved, but still delete the first row.) The corresponding integral vector, whose Ferrers diagram is $F^{j \mid}$, is denoted by $a^{j \mid}$ and this vector lies in $\mathbb{R}^{n-1}$. We call $a^{j \mid}$ a splitting of $a$.

Example 3.1. Let $a=(6,5,3,2,2)$ and $j=4$. Then $a^{4}=(4,4,4,2)$. The Ferrers diagram of $a$ and $a^{4 \mid}$ are shown below

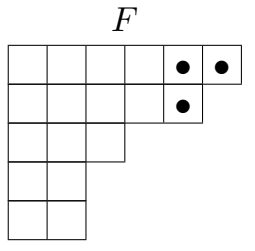

$a=(6,5,3,2,2)$

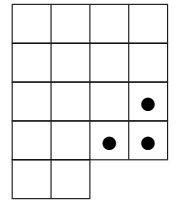

$F^{4}$

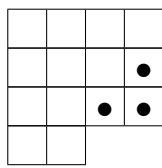

$a^{4}=(4,4,4,2)$

Bullets indicate the boxes that were moved, and the intermediate Ferrers diagram (before the first row was deleted) is also shown.

For fixed $j$ with $\lceil N / n\rceil \leq j \leq a_{1}$, we can give an explicit expression for $a^{j \mid}$. Define $\bar{a}_{1: s}=\frac{1}{s} \sum_{i=1}^{s} a_{i} \quad(1 \leq s \leq n)$ which is the (arithmetic) mean of the first $s$ components of $a$. Since $a$ is monotone,

$$
a_{1}=\bar{a}_{1: 1} \geq \bar{a}_{1: 2} \geq \cdots \geq \bar{a}_{1: n}=\frac{1}{n} \sum_{i=1}^{n} a_{i}=\frac{N}{n} .
$$

Now let $q(1 \leq q \leq n)$ be the largest integer such that $\bar{a}_{1: q} \geq j$; such $q$ exists and is unique (and depends on $j$ ). The $i$ th component $a_{i}^{j \mid}$ of the vector $a^{j \mid} \in \mathbb{R}^{n-1}$ is given by

$$
a_{i}^{j \mid}=\left\{\begin{array}{cl}
j & (1 \leq i \leq q-1) \\
\sum_{t=1}^{q+1} a_{t}-q j & (i=q) \\
a_{i+1} & (q+1 \leq i \leq n-1)
\end{array}\right.
$$


that is, written out explicitly,

$$
a^{j \mid}=\left(j, \ldots, j, \sum_{t=1}^{q+1} a_{t}-q j, a_{q+1}, \ldots, a_{n}\right) \in \mathbb{R}^{n-1} .
$$

One may verify that $a^{j \mid}$ is monotone and $\sum_{i=1}^{n-1} a_{i}^{j \mid}=N-j$.

The construction of $a^{j \mid}$ leads to the following proposition concerning splittings. It also gives a recursive expression for the counting function $\nu^{*}$.

Proposition 3.2. Let $x=\left(x_{1}, x_{2}, \ldots, x_{n}\right)$ and $a=\left(a_{1}, a_{2}, \ldots, a_{n}\right)$ be monotone integral vectors in $\mathbb{R}^{n}$. Then $x \preceq a$ if and only if $\left(x_{2}, x_{3}, \ldots, x_{n}\right) \preceq a^{x_{1} \mid}$. Moreover, with $N=\sum_{j=1}^{n} a_{j}$, we have

$$
\nu^{*}(a)=\sum_{j=\lceil N / n\rceil}^{a_{1}} \nu^{*}\left(a^{j \mid}\right)
$$

Proof. By definition, $x \in M_{I}^{*}(a)$ means that $x$ is (integral) monotone and majorized by $a$. Considering the vectors in $M_{I}^{*}(a)$ with the first component being $j$ and from the construction of the Ferrers diagram of $a^{j \mid}$ (with moved boxes in the topmost rows), we see that the set $\left\{x \in M_{I}^{*}(a): x_{1}=j\right\}$ is the same as

$$
\left\{x \in \mathbb{Z}^{n}: x \text { is monotone, } x_{1}=j,\left(x_{2}, x_{3}, \ldots, x_{n}\right) \preceq a^{j \mid}\right\} .
$$

(Here $\mathbb{Z}^{n}$ for integral vectors in $\mathbb{R}^{n}$.) This proves the first statement of the theorem.

Next, note that every vector $x$ in $M_{I}^{*}(a)$ satisfies $x_{1} \geq\lceil N / n\rceil$ (due to monotonicity and $\left.\sum_{j=1}^{n} x_{j}=N\right)$. We count $M_{I}^{*}(a)$ by partitioning this set according to the value of the first component of its vectors. Thus, by the first part of this theorem, we have

$$
\begin{aligned}
\nu^{*}(a) & =\sum_{j=\lceil N / n\rceil}^{a_{1}}\left|\left\{x \in M_{I}^{*}(a): x_{1}=j\right\}\right| \\
& =\sum_{j=\lceil N / n\rceil}^{a_{1}} \mid\left\{(j, z) \in \mathbb{Z} \times \mathbb{Z}^{n-1}:(j, z) \text { is monotone, } z \preceq a^{j \mid}\right\} \mid \\
& =\sum_{j=\lceil N / n\rceil}^{a_{1}}\left|M_{I}^{*}\left(a^{j \mid}\right)\right| \\
& =\sum_{j=\lceil N / n\rceil}^{a_{1}} \nu^{*}\left(a^{j \mid}\right),
\end{aligned}
$$

so (3.2) holds.

Equation (3.2) in Proposition 9 clearly gives an algorithm for computing $\nu^{*}(a)$. Combined with the formula in Proposition 2.4, this algorithm may also be used to compute $\nu(a)$. Note that in (3.2) the computation of $\nu^{*}\left(a^{j \mid}\right)$ can sometimes be simplified, especially for "small" $j$, by using the property $\nu^{*}(b)=\nu^{*}\left(b-b_{n} e\right)$ for a monotone vector $b$ (where $e$ is the all-ones vector and $b_{n}$ is the smallest component of $b$ ), see Proposition 2.3. Proposition 3.2 leads to an algorithm for enumeration of the set $M_{I}^{*}(a)$ : compute the $a^{j \mid}$ 's, and then repeat this process for each of the constructed $a^{j \mid}$ 's, etc. 
Example 3.1 - continued. Consider again $a=(6,5,3,2,2)$. So $n=5, N=18$ and $\lceil N / n\rceil=4$. The Ferrers diagram of $F^{j \mid}$ for $j=4,5,6$ are
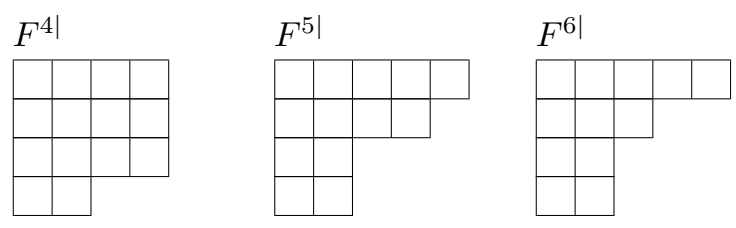

$$
a^{4 \mid}=(4,4,4,2) \quad a^{5 \mid}=(5,4,2,2) \quad a^{6 \mid}=(5,3,2,2)
$$

Using formula (3.2) recursively, we compute

$$
\nu^{*}\left(a^{4 \mid}\right)=2, \quad \nu^{*}\left(a^{5}\right)=4, \quad \nu^{*}\left(a^{6 \mid}\right)=4 .
$$

Therefore, by $(3.2), \nu^{*}(a)=2+4+4=10$.

Let $a=\left(a_{1}, a_{2}, \ldots, a_{n}\right) \in \mathbb{R}^{n}$ be a nonnegative integral monotone vector, and define $m=a_{1}$. The conjugate of $a$ is the vector $a^{*}=\left(a_{1}^{*}, a_{2}^{*}, \ldots, a_{m}^{*}\right) \in \mathbb{R}^{m}$, where

$$
a_{k}^{*}=\left|\left\{i: a_{i} \geq k\right\}\right| \quad(1 \leq k \leq m) .
$$

If $F$ is the Ferrers diagram corresponding to $a$, the row sums in $F$ (viewing boxes as ones, and otherwise having zeros) are the components in $a$ while the column sums are the components in $a^{*}$. In particular, the Ferrers diagram of $a^{*}$ is the transpose of $F$ (making rows into columns, as for matrices). For instance, if $a=(2,2,2,1,1)$, then $a^{*}=(5,3)$.

Proposition 3.3. Let $a=\left(a_{1}, a_{2}, \ldots, a_{n}\right) \in \mathbb{R}^{n}$ be a nonnegative monotone integral vector. Then

$$
\nu(a) \leq \prod_{k=1}^{a_{1}}\left(\begin{array}{c}
n \\
a_{k}^{*}
\end{array}\right) .
$$

Equality holds if and only if $a=(s+1, \ldots, s+1, s, \ldots, s)$ for some $s$.

Proof. Let $\xi_{k}=(1, \ldots, 1,0, \ldots, 0) \in \mathbb{R}^{n}$ be the vector with $k$ leading ones and otherwise zeros $(k \leq n)$. We decompose $a=\left(a_{1}, a_{2}, \ldots, a_{n}\right)$ as

$$
a=\sum_{k=1}^{m} \xi_{a_{k}^{*}} \quad\left(m=a_{1}\right) .
$$

By Proposition 2.3 (iii) and Corollary 2.6, we have

$$
\nu(a)=\nu\left(\sum_{k=1}^{m} \xi_{a_{k}^{*}}\right) \leq \prod_{k=1}^{m} \nu\left(\xi_{a_{k}^{*}}\right)=\prod_{k=1}^{m}\left(\begin{array}{c}
n \\
a_{k}^{*}
\end{array}\right) .
$$

Equality in (3.3) occurs if and only if overall equality in (3.4) holds, which is true, by Proposition 2.3 (iii), if and only if one of $\xi_{a_{k}^{*}}$ 's is non-constant. By Corollary 2.6, $a$ is of the desired form. 
Example 3.4. Let $a=(2, \ldots, 2,1, \ldots 1) \in \mathbb{R}^{n}$, in which the number of 2 's is $k$. Then $a^{*}=(n, k)$. So Proposition 3.3 gives

$$
\nu(a) \leq\left(\begin{array}{l}
n \\
n
\end{array}\right) \cdot\left(\begin{array}{l}
n \\
k
\end{array}\right)=\left(\begin{array}{l}
n \\
k
\end{array}\right)
$$

which we know (by Proposition 2.6) is tight, i.e., $\nu(a)=\left(\begin{array}{l}n \\ k\end{array}\right)$. If $a=(4,2,1)($ Example 2.1), then $a^{*}=(3,2,1,1)$ and $\nu(a)=12$ while the bound in Proposition 3.3 is $1 \cdot 3 \cdot 3 \cdot 3=27$.

As this example shows, the quality of the bounds we have found is highly dependent on vector $a$ itself. We believe that the bound in Proposition 3.3 may be acceptable when "the span" $a_{1}-a_{n}$ is rather small.

\section{Acknowledgments}

This work was partially supported by an NSU FCAS Mini-grant.

\section{References}

[1] G.E. Andrews, The Theory of Partitions (Cambridge University Press 1998).

[2] A. Barvinok, A Course in Convexity (Graduate Studies in Math. 54, AMS 2002).

[3] R.A. Brualdi, Combinatorial Matrix Classes (Cambridge University Press 2006).

[4] T. Brylawski, The lattice of integer partitions, Discrete Math. 6 (1973) 201-209.

[5] G. Dahl, Principal majorization ideals and optimization, Linear Algebra Appl. 331 (2001) 113-130.

[6] G. Dahl, Majorization permutahedra and (0,1)-matrices, Linear Algebra Appl. 432 (2010) 3265-3271.

[7] G.H. Hardy, J.E. Littlewood and G. Pólya, Inequalities (Cambridge University Press, Reprint 1994).

[8] A.W. Marshall, I. Olkin and B. Arnold, Inequalities: Theory of Majorization and Its Applications (2nd ed., Springer 2011).

[9] J.H. van Lint and R.M. Wilson, A Course in Combinatorics (2nd ed., Cambridge University Press 2001).

[10] S. Onn and E. Vallejo, Permutohedra and minimal matrices, Linear Algebra Appl. 412 (2006) 471-489.

[11] R.P. Stanley, Enumerative Combinatorics (Vol.1, 2nd ed., Cambridge University Press 2011).

[12] H.S. Wilf, Lectures on Integer Partitions (lecture notes, University of Pennsylvania 2000).

[13] G. Ziegler, Lectures on Polytopes (Springer 1995).

[14] F. Zhang, Matrix Theory: Basic Results and Techniques (2nd ed., Springer 2011). 